\title{
CONSIDERAÇÕES PRÁTICAS SOBRE CONTROLE AVANÇADO DE PROCESSO APLICADO A MOINHO DE BOLAS
}

\author{
R. GEDRAITE ${ }^{1}$, S. M. S. NEIRO ${ }^{1}$, A. S. MORAIS ${ }^{1}$, L. VIEIRA ${ }^{2}$, L. A. RODRIGUES ${ }^{2}$ \\ ${ }^{1}$ Faculdade de Engenharia Química - Universidade Federal de Uberlândia \\ ${ }^{2}$ Honeywell Process Solutions \\ rgedraite@feq.ufu.br; sergioneiro@feq.ufu.br; aniel@eletrica.ufu.br;
}

Artigo submetido em novembro/2013 e aceito em maio/2014

DOI: http://dx.doi.org/10.15628/holos.2014.1764

\section{RESUMO}

A aplicação de estratégia de controle avançado em moinhos de bolas tipicamente usados em usinas de beneficiamento de minério é um tema cuja importância vem crescendo sistematicamente. Tal fato é justificado pelo aumento de produção, melhoria da qualidade do produto final, economia de energia e menor desgaste do revestimento interno do moinho. O sistema de controle avançado apresentado neste trabalho contempla o emprego de controlador multivariável acoplado a sistema de controle regulatório, que permite ao moinho alcançar condições ótimas de operação de acordo com os critérios técnicos estabelecidos como premissa de projeto. A implantação do sistema de controle avançado foi realizada de forma simples e rápida, permitindo comunicação direta com o sistema de controle regulatório (SDCD/CLP). Os resultados obtidos permitem afirmar que houve significativa redução da variabilidade na granulometria do material após a moagem, redução do consumo de energia e aumento de produção.

PALAVRAS-CHAVE: Controle multivariável, moinhos de bolas, variabilidade

\section{PRATICAL CONSIDERATIONS ABOUT ADVANCED PROCESS CONTROL APPLIED TO BALL MILLS}

\begin{abstract}
The application of advanced control strategy for ball mills typically used in ore beneficiation plants is a topic whose importance has been growing systematically. This fact is justified by increasing production, improving quality of final product, energy savings and integrity of the mill shell and drive components. The advanced control system presented in this paper considers the use of multivariable controller coupled to regulatory control system, which allows the mill to achieve optimum operation conditions
\end{abstract}

according to the technical criteria set premised project. The implementation of advanced control system was carried out quickly and easily, enabling direct communication with the regulatory control system (DCS/PLC). The results obtained suggest a significant reduction of variability in material particle size distribution after grinding, reducing energy consumption and increasing production.

KEYWORDS: Multivariable control; ball mills; variability. 


\section{INTRODUÇÃO}

As aplicações dos moinhos de bolas estão presentes na grande maioria das indústrias de mineração e metalurgia. Este equipamento é tipicamente caracterizado pela baixa eficiência energética, decorrente da própria natureza do processo de fragmentação. O custo relacionado à redução no tamanho das partículas aumenta conforme o grau de fragmentação. Por exemplo, a operação de britagem pode consumir até $1 \mathrm{kWh} / \mathrm{t}$ ao passo que a operação de moagem pode consumir $10 \mathrm{kWh} / \mathrm{t}$. Tipicamente,o consumo de energia nesta operação unitária pode representar de 30 a 50\% do consumo global de energia em uma planta. Em alguns casos como, por exemplo, no processamento de material muito duro ou quando se deseja obter um produto final ultrafino, o consumo de energia pode chegar a 70\% (JKMRC, 1995).

Poucos trabalhos na literatura apresentam resultados práticos obtidos com a implantação de sistemas de controle avançado em processos industriais. A razão para esta lacuna reside muito provavelmente na dificuldade de se obter autorização para divulgar dados operacionais dos processos estudados. GOUVEIA et al. (2009) e RESTREPO et al. (2009) analisaram a aplicação de sistemas de controle avançado em diferentes plantas industriais, com foco na análise da redução da variabilidade obtida e no aumento de produção e/ou economia de energia.

Os algoritmos de Controle Preditivo baseados em Modelo (MPC, em inglês) têm sido amplamente estudados e aplicados na indústria de mineração em anos recentes, devido fundamentalmente à sua capacidade para tratar processos multivariáveis que possuem restrições. O sucesso dos algoritmos MPC pode ser comprovado pela grande variedade de controladores preditivos existentes no mercado. SEBORG et al. (2011) afirmam que de uma maneira geral, nos diferentes segmentos industriais existem centenas ou até milhares de controladores preditivos.

O objetivo específico de controle do sistema de controle avançado de processo (APC, em inglês) aplicado no moinho de bolas estudado neste trabalho foi operar o equipamento de maneira estável e segura, produzindo partículas com tamanho desejado, na máxima taxa de produção préestabelecida e com a máxima eficiência, sem, contudo permitir que ocorra a violação das limitações de processo que nele também se encontram configuradas.

\section{OPERAÇÃO DE MOAGEM VIA ÚMIDA EM CIRCUITO FECHADO}

Na operação via úmida, a adição de água no circuito de processamento de um moinho de bolas é tipicamente utilizada para controlar os seguintes parâmetros do processo: (i)- a carga do moinho, (ii)- a porcentagem de sólidos na corrente de saída, (iii)- o nível de polpa na caixa de bombas e (iv)- a granulometria na corrente de overflow da seção de hidrociclonagem. A redução da porcentagem de sólidos na corrente de saída favorece o aumento na eficiência da operação de moagem, proporcionando uma redução considerável no consumo de bolas. Porém, esse aumento de eficiência tem um ponto crítico a partir do qual a mesma começa a diminuir, pois a polpa alimentada com baixa porcentagem de sólidos reduz as colisões entre as bolas e as partículas. Isto ocorre por que as partículas sólidas tendem a se dispersar na polpa e o resultado é o aumento das colisões entre as próprias bolas e entre estas e o revestimento, implicando no aumento no 
desgaste do revestimento associado ao aumento no consumo de bolas. A Figura 1 apresenta uma representação simplificada de um processo de moagem via úmida em circuito fechado.

A polpa retirada do moinho de bolas é transferida para a caixa de bombas, onde é misturada com a carga circulante proveniente da corrente de underflow da seção de hidrociclonagem e da linha de polpa da bomba de piso. O nível da polpa é medido por meio de sensor de nível ultrassônico, instalado na caixa de bombas, o qual é responsável por fornecer sinal continuo de nível de material. Um inversor de frequência é utilizado como elemento final de controle, o qual é responsável tanto pelo ajuste na velocidade de rotação das bombas como por manter constante a pressão da polpa enviada para o sistema de classificação e o nível da caixa de bombas.

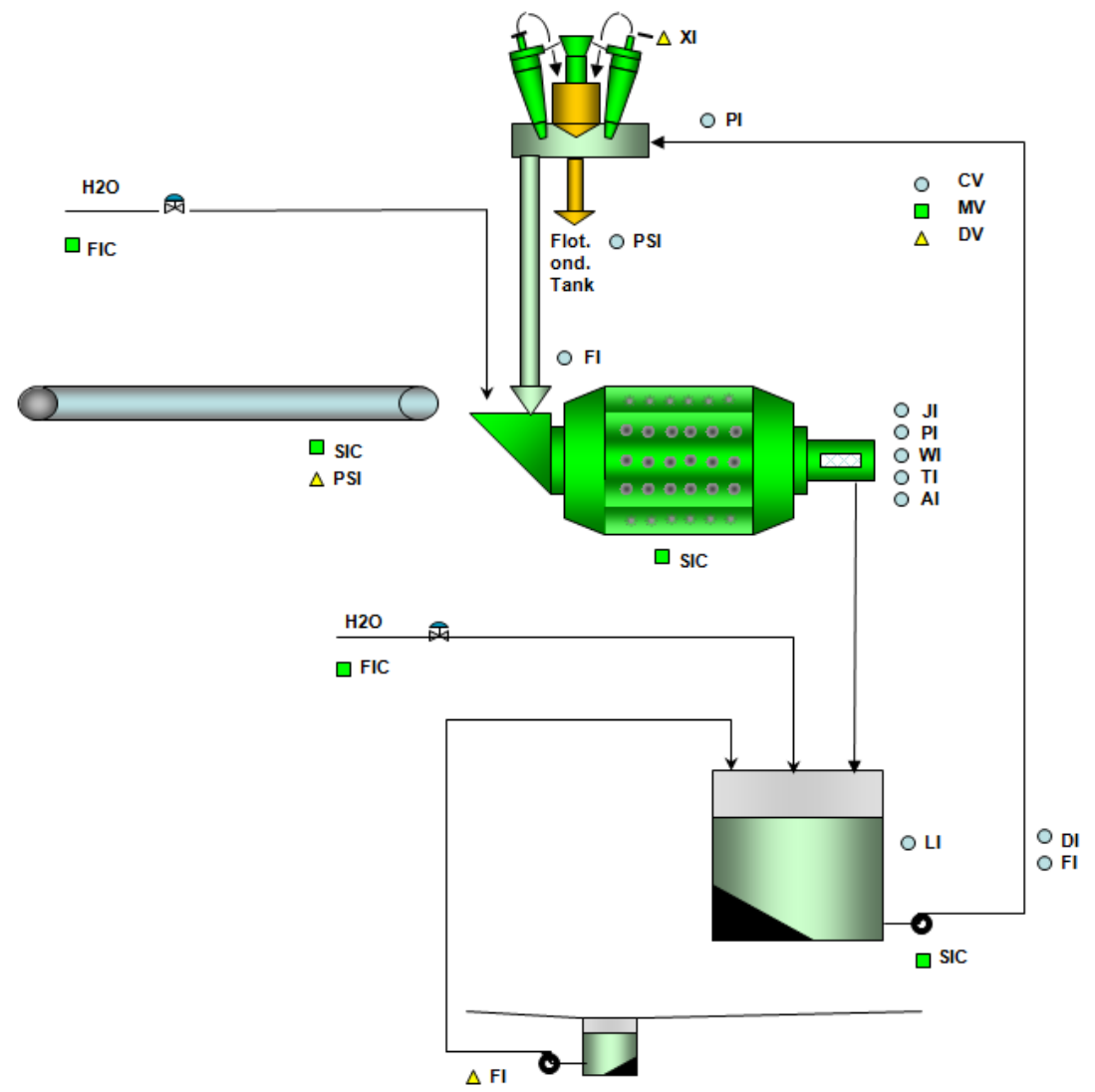

Figura 1 - Representação do Processo de Moagem via úmida em circuito fechado.

Os principais parâmetros que devem ser ajustados para controlar o tamanho das partículas no overflow da bateria de hidrociclones são: (i)- porcentagem de sólidos na polpa de alimentação; (ii)- distribuição granulométrica da alimentação e (iii)- pressão de alimentação da bateria de hidrociclones (RAMASAMY, NARAYANAN \& RAO, 2005).

O princípio fundamental da estratégia de controle está baseado no fato de que tipicamente a produção máxima é obtida com a potência máxima do motor. O ponto de potência máxima depende das características da corrente de alimentação do moinho, das condições do revestimento, e do nível de enchimento dos corpos moedores.

Quando um moinho opera com sobrecarga, a carga do moinho aumenta, mas a potência é reduzida, porque o material não esta sofrendo o efeito da tombagem. Quando isso acontece, 
material começa a acumular no interior do moinho e o efeito de centrifugação provoca a redução da eficiência, o aumento do desgaste do revestimento e do consumo de bolas.

\section{CONSIDERAÇÕES SOBRE O EMPREGO DE SISTEMA DE CONTROLE AVANÇADO APLICADO AO MOINHO DE BOLAS EM ESTUDO}

O objetivo geral de um APC é auxiliar o operador de processos na operação da unidade industrial sob condições ótimas. O APC alcança este objetivo operando em conjunto com o sistema de controle regulatório. Por este motivo, torna-se essencial que, tanto a instrumentação de processo como as malhas de controle regulatório estejam em condições adequadas e estáveis de funcionamento para que o APC atue apropriadamente. Inicialmente, é necessário estabilizar o processo para posteriormente realizar a otimização econômica, ou seja, a melhoria no desempenho do processo é obtida através da redução na variabilidade das principais variáveis. $\mathrm{Na}$ Figura 2 são apresentados, na forma de diagrama de blocos, os principais subsistemas que compõem o sistema de controle avançado em estudo.

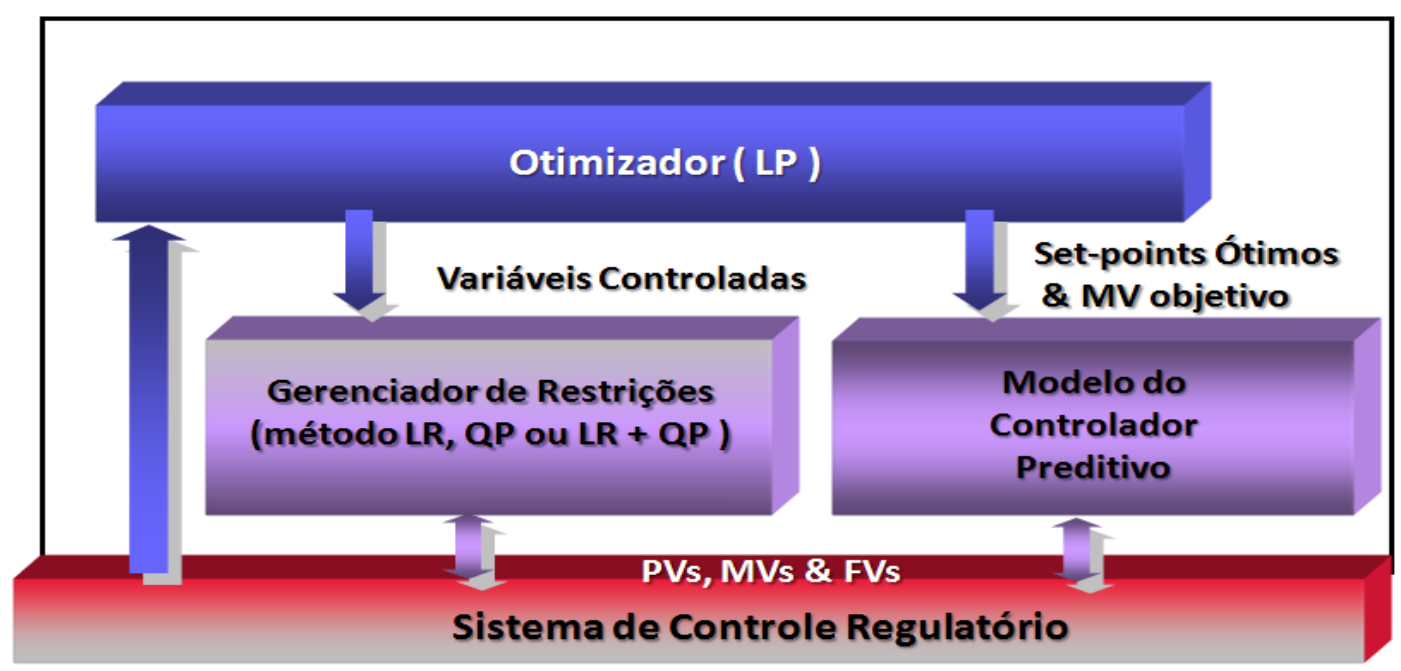

Figura 2 - Principais elementos do sistema de controle avançado em estudo.

O pacote de otimização e de controle multivariável utilizado neste trabalho executou não só o controle preditivo multivariável como também é dotado de um otimizador linear (LP), que permite ao sistema atingir as condições de operação ótimas e econômicas, respeitando às restrições determinadas pelo operador do processo. O Otimizador Econômico utiliza o mesmo modelo de processo utilizado pelo MPC para realizar as predições de estado estável e determinar as metas a serem alcançadas. Isto é completamente transparente para o operador.

A configuração do Otimizador Econômico requer que se estabeleçam custos e benefícios relativos para as variáveis que determinam a eficiência e rentabilidade do processo. As variáveis consideradas neste trabalho foram: (i)- alimentação de minério, considerada como benefício e (ii)consumo de energia, considerada como custo.

Com base nos valores preditos correspondentes ao estado estacionário, o controlador multivariável minimiza a função objetivo visando manter todas as variáveis controladas (CVs) e 
variáveis manipuladas (MVs) nos respectivos limites. O MPC atua simultaneamente sobre as variáveis controladas (CVs) e conduz o equipamento a seu ponto ótimo de funcionamento, compreendido entre os seus limites de operação e segurança, enviando set-points para as variáveis manipuladas (MVs) em consonância com os respectivos limites máximos e mínimos especificados. O MPC utiliza um conjunto de modelos pré-determinados de modo a predizer os valores futuros das variáveis de controle e as correspondentes alterações a serem aplicadas às variáveis manipuladas. O MPC também monitora a variação sofrida pelas variáveis feedforward (FFs), buscando compensar os seus efeitos (QIN \& BADGWELL, 2003).

O sistema de controle avançado em estudo define o set-point dos seguintes controladores PID que compõem a base do sistema de controle regulatório: (i)- alimentação de minério fresco, (ii)- vazão de água alimentada ao moinho, (iii)- vazão de água alimentada à caixa de bombas e (iv)velocidade de rotação da bomba, respeitando as seguintes restrições: (i)- limites de potência total, (ii)- a pressão média nos apoios, (iii)- nível na caixa de bombas, (iv)- pressão na bateria de hidrociclones e (v)- percentual de partículas retida na malha de abertura 65. Na Figura 3 é apresentada a visão geral da estrutura do controlador empregado no sistema de controle avançado do processo.

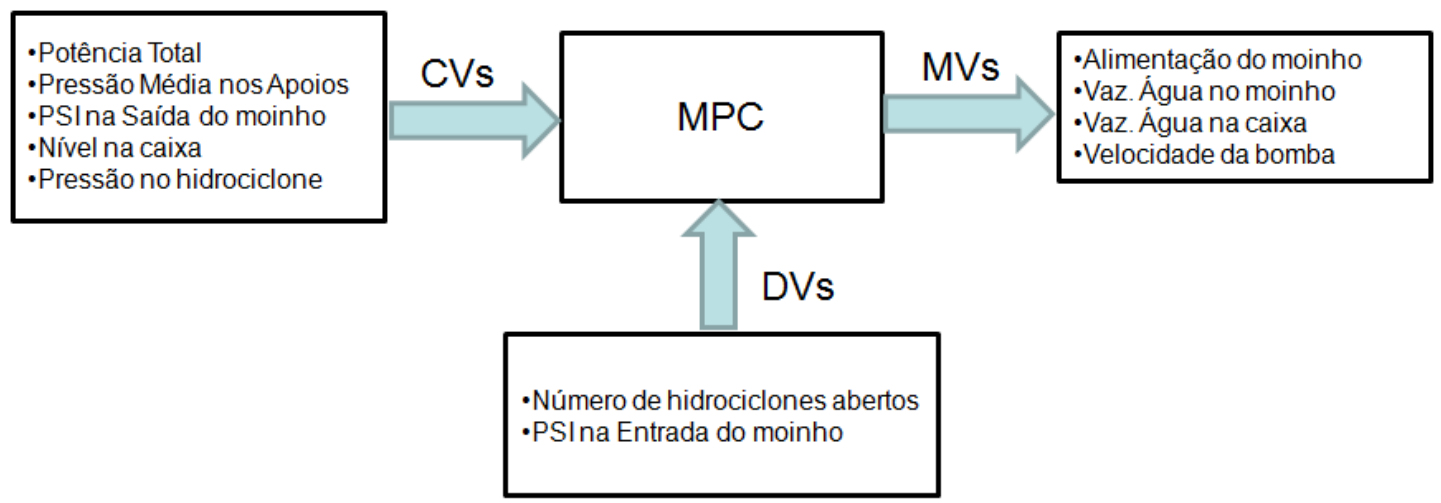

Figura 3 - Estrutura do controlador empregado.

Adicionalmente, o sistema de controle avançado em estudo é responsável por ajustar as variáveis manipuladas de tal forma que o moinho opere no limite superior de potência total determinado pelo operador. Caso alguma restrição de processo diferente seja verificada, tal como a ocorrência de pressão média elevada nos apoios, o APC poderá executar uma ou mais das seguintes ações: (i)- redução na vazão de alimentação de minério fresco, e (ii)- aumento da vazão de água para o moinho. A qualquer momento, o operador do processo poderá desligar parcial ou totalmente o sistema de controle avançado, ou ainda manter o mesmo em operação dentro de limites pré-estabelecidos (LAAKSONEN \& PYöTSIä, 1998).

O nível na caixa de bombas, a potência total consumida, a pressão nos apoios, a pressão de alimentação dos hidrociclones e a velocidade angular do rotor da bomba de alimentação de água foram configuradas como variáveis de controle restritivas. Trata-se do caso das variáveis que podem ou não estar ativas entre os valores definidos como limites de alto e baixo, atribuídos pelo operador, em condições normais de operação. Ao exceder estes limites ou mesmo se o MPC 
predizer que estes poderão ser excedidos, estas variáveis passam a ser consideradas (caso estejam ativas) e o MPC tenta controlá-las de forma a mantê-las no intervalo definido pelos seus limites.

Considerando a dificuldade de medição direta das variáveis associadas à qualidade do produto, o uso de sensor virtual foi considerado para a inferência em tempo real da distribuição do tamanho das partículas (HONEYWELL, 2008).

Os modelos do processo foram obtidos usando a abordagem FOPDT (First order plus dead time), a qual é largamente usada para representar a dinâmica de processos industriais (AGUIRRE, 2007). Os parâmetros dos modelos, $K_{P}$ (ganho do processo), $\tau_{P}$ (constante de tempo do processo) e $\theta_{p}$ (atraso de transporte do processo) foram calculados com base no método da curva de resposta a uma mudança em degrau. Na Figura 4 é apresentada uma representação simplificada da obtenção dos modelos semi-empíricos considerados neste trabalho.

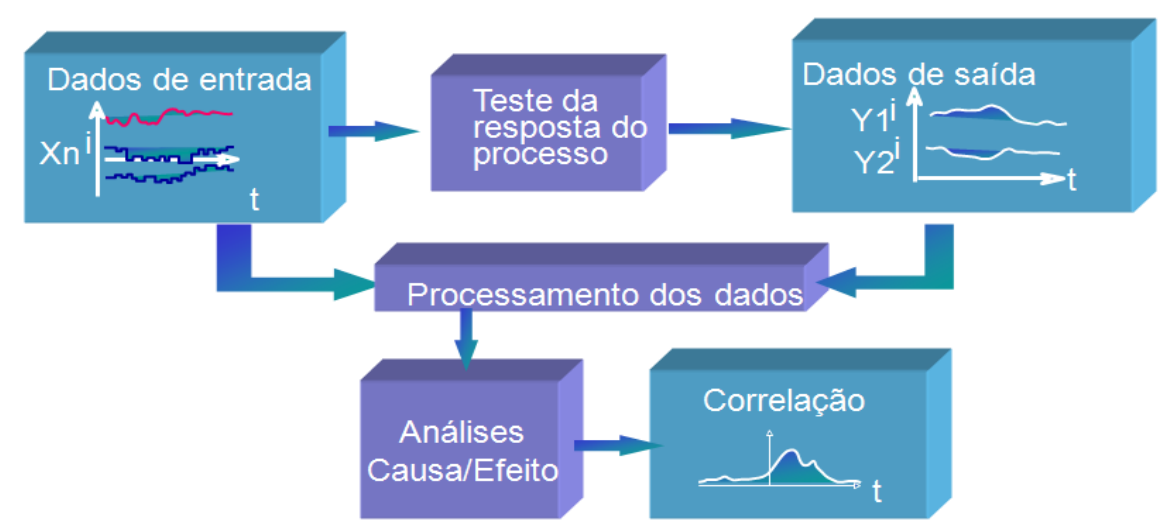

Figura 4 - Representação do Processo de obtenção dos modelos semi-empíricos.

As variáveis controladas utilizadas bem como seus objetivos de controle são apresentadas na Tabela 1. Quando o processo requer que a variável controlada esteja compreendida entre os limites máximos e mínimos, a tabela mostrará MIN/MAX. Se este limite estiver restrito a apenas um dos extremos a tabela indicará MAX ou MIN. Nos casos em que houver necessidade de que a variável controlada persiga uma meta especificada, a tabela indicará SP. As variáveis manipuladas e as variáveis do tipo feedforward estão listadas na Tabela 2.

Um conjunto de telas pré-configuradas na tela de operação do APC permite ao operador do moinho obter informações sobre o estado do APC, bem como o acesso aos parâmetros operacionais necessários.

O APC foi instalado em um microcomputador do tipo PC conectado à rede industrial. A comunicação entre o sistema de controle avançado e o sistema digital de controle distribuído foi realizada por meio do protocolo OPC.

O Controle Supervisório Seguro (SSC, em inglês) permite que o APC execute o controle supervisório do processo com base em blocos de controle residentes nas estações de controle do sistema digital de controle distribuído. O SSC garante que o set-point do computador supervisório não será utilizado, a menos que seja validado. O SSC foi utilizado por todas as malhas de controle regulatório que recebem o set-point do sistema de controle avançado. 
Tabela 1 - Variáveis controladas.

\begin{tabular}{c|c}
\hline VARIÁVEL CONTROLADA & OBJETIVO DE CONTROLE \\
\hline Potência Total (KW/h) & MAX \\
\hline Pressão Média nos Apoios (psi) & MIN/MAX \\
\hline PSD - na saída do moinho (\%) & MIN/MAX \\
\hline Nível na Caixa de Bombas (\%) & MIN/MAX \\
\hline Pressão no hidrociclone (bar) & MAX \\
\hline
\end{tabular}

Fonte - Honeywell Process Solutions.

Tabela 2 - Variáveis manipuladas e feedforward.

\begin{tabular}{c|c}
\hline VARIÁVEIS MANIPULADAS & VARIÁVEIS FEEDFORWARD \\
\hline Alimentação do moinho (ton $/ \mathrm{h})$ & Número de Hidrociclones \\
\hline Vaz. de água $\mathrm{p} /$ o moinho $\left(\mathrm{m}^{3} / \mathrm{h}\right)$ & PSD - na entrada do moinho $(\%)$ \\
\hline Vaz. de água $\mathrm{p} /$ caixa de bombas $\left(\mathrm{m}^{3} / \mathrm{h}\right)$ & \\
\hline Vel. da bomba de água (RPM) & \\
\hline
\end{tabular}

Fonte - Honeywell Process Solutions.

\section{RESULTADOS OBTIDOS E DISCUSSÃO}

Os resultados experimentais obtidos com a implantação da estratégia de controle avançado apresentados neste trabalho foram obtidos com base em análises estatísticas dos resultados experimentais coletados por períodos de tempo suficientemente amplos, utilizando o aplicativo MINITAB ${ }^{\circledR}$. Os dados foram coletados na instalação industrial estudada, utilizando a ferramenta para armazenamento de dados históricos (PIMS) existente no sistema de controle.

Os resultados experimentais obtidos com a implantação da estratégia de controle avançado apresentados neste trabalho foram obtidos com base em análises estatísticas dos resultados experimentais coletados por períodos de tempo suficientemente amplos, utilizando o aplicativo MINITAB ${ }^{\circledR}$. Os dados foram coletados na instalação industrial estudada, utilizando a ferramenta para armazenamento de dados históricos (PIMS) existente no sistema de controle.

A análise comparou 3 meses de operação com APC e 3 meses de operação sem APC. Nas Figuras 5, 6 e 7 são apresentados os valores da média, do desvio padrão e a distribuição normal para as variáveis de processo consideradas como indicadores de desempenho do moinho, a saber: consumo específico de energia, vazão de alimentação e percentagem de partículas com diâmetro fora de especificação. 


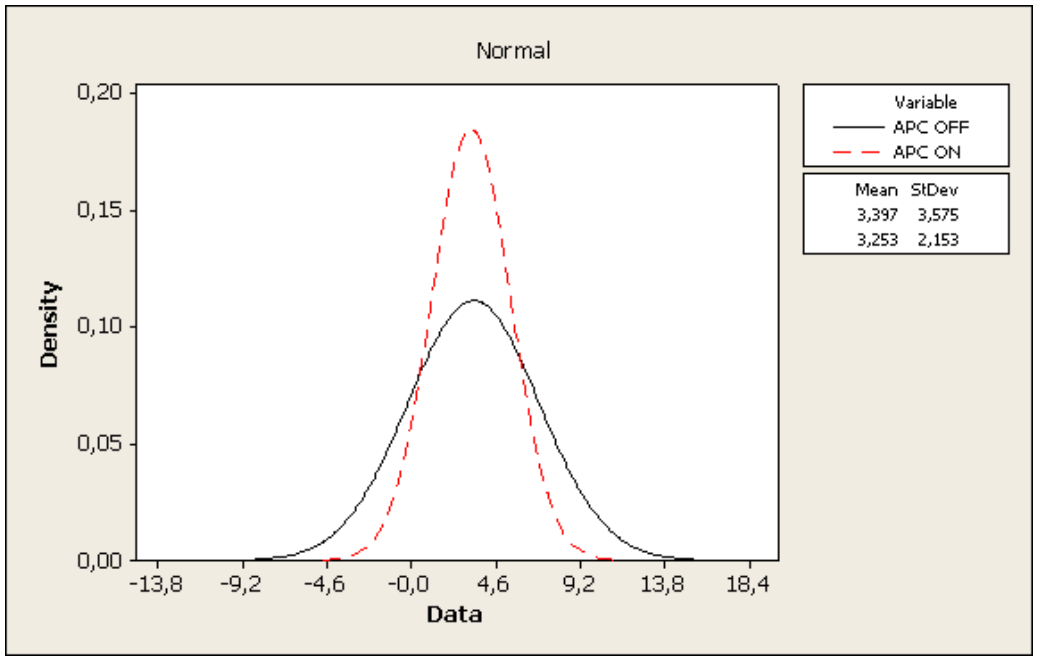

Figura 5 - Consumo específico de energia (kWh/ton).

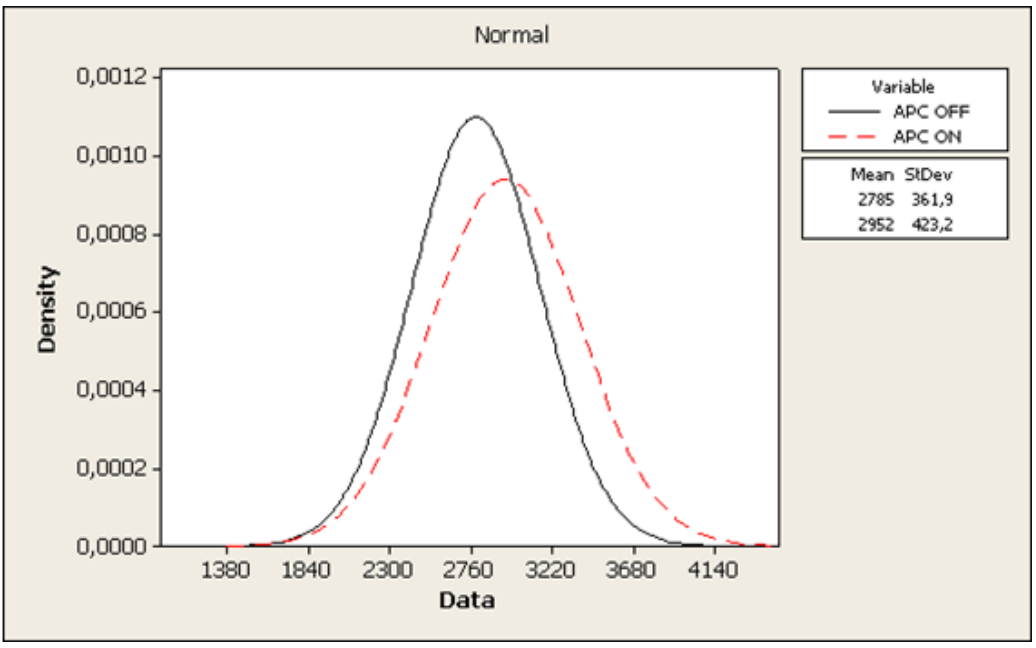

Figura 6 - Vazão de alimentação do moinho (ton/h).

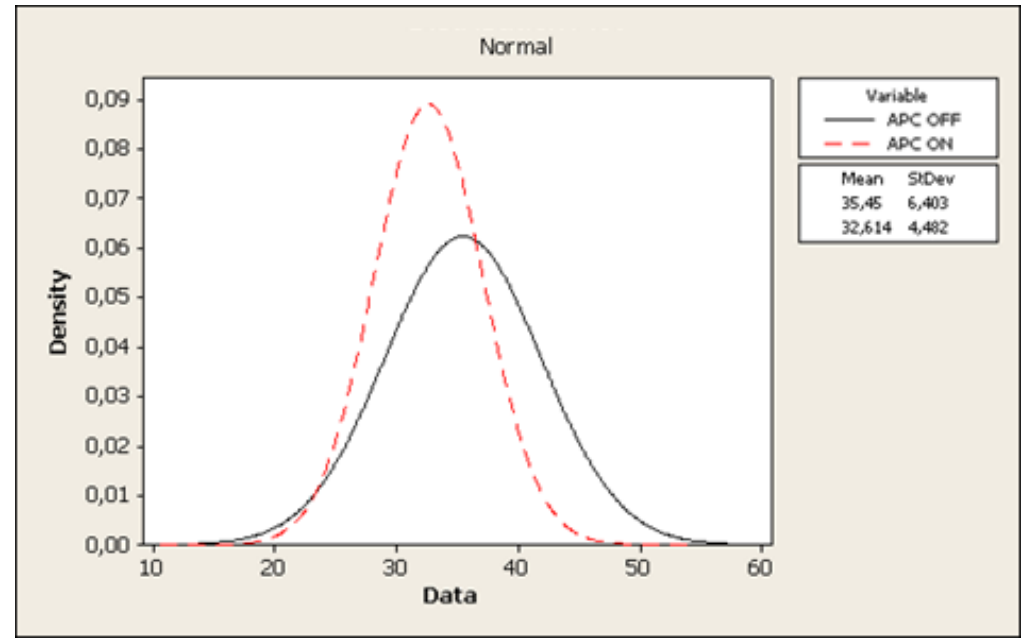

Figura 7 - Porcentagem de partículas com tamanho fora da especificação (\%). 
Existem diferentes maneiras de se quantificar a variabilidade apresentada pelo processo, a saber (LAAKSONEN \& PYöTSIä (1998); McMILLAN (1994)):

1. Medir a máxima variabilidade do sinal de saída do processo;

2. Calcular ao longo do tempo a área do erro entre o valor de referência e a variável medida, usando técnicas IAE - Integrated Absolute Error ou ISE - Integrated Square Error;

3. Duplicar o valor do desvio padrão do erro $(\sigma)$ e dividi-lo pelo valor médio da saída do processo $(\mu)$, isto é, calculando $(2 \cdot \sigma / \mu)$; ou ainda,

4. Duplicar o valor do desvio padrão do erro $(2 \cdot \sigma)$.

O critério adotado para avaliar a variabilidade apresentada pelas variáveis de processo consideradas neste trabalho baseou-se no critério de duplicar o desvio padrão e dividir o valor obtido pelo valor da média. A Tabela 3 apresenta os resultados obtidos para a variabilidade.

Tabela 3 - Resultados obtidos para a variabilidade.

\begin{tabular}{c|c|c}
\hline VARIÁVEL ANALISADA & SEM APC & COM APC \\
\hline Consumo específico de energia & 2,10 & 1,32 \\
\hline Alimentação do moinho & 0,26 & 0,29 \\
\hline $\begin{array}{c}\text { Porcentagem de partículas com tamanho fora da } \\
\text { especificação }\end{array}$ & 0,36 & 0,27 \\
\hline
\end{tabular}

Fonte - Honeywell Process Solutions.

Os resultados obtidos mostram o aumento na produção, a redução no consumo específico de energia e a melhoria na qualidade do produto final obtidos com o emprego do APC. Assim sendo, para o consumo específico de energia, notou-se uma redução de aproximadamente $4 \%$, ao passo que para a vazão de alimentação do moinho, o aumento foi de aproximadamente $6 \%$. A redução na quantidade de partículas produzidas com tamanho fora de especificação foi de aproximadamente $8 \%$. Complementarmente, a redução da variabilidade para o consumo específico de energia foi de aproximadamente $37,1 \%$, determinada com base nas informações apresentadas na Tabela 3. Já para o aumento de vazão de alimentação do moinho, foi verificado um aumento no valor da variabilidade de aproximadamente $11,5 \%$. A possível justificativa para este comportamento pode estar relacionada com o fato de o controlador MPC atuar de forma mais frequente sobre a vazão de alimentação do moinho, buscando minimizar o consumo específico de energia. A redução de variabilidade na quantidade de partículas produzidas com tamanho fora de especificação foi de $25 \%$. É importante ressaltar o fato de que as melhorias anteriormente citadas foram obtidas devido à atuação do APC, que consegue executar o gerenciamento das restrições de maneira ótima.

O aumento da potência de trabalho do moinho reflete diretamente na eficiência da operação de moagem e consequentemente, no consumo específico de energia como mostram as Figuras 8 e 9. Com o emprego do APC pode-se verificar que os valores da potencia do moinho medida experimentalmente ficaram mais próximas da curva de operação do equipamento, sugerindo melhor rendimento operacional do mesmo. 


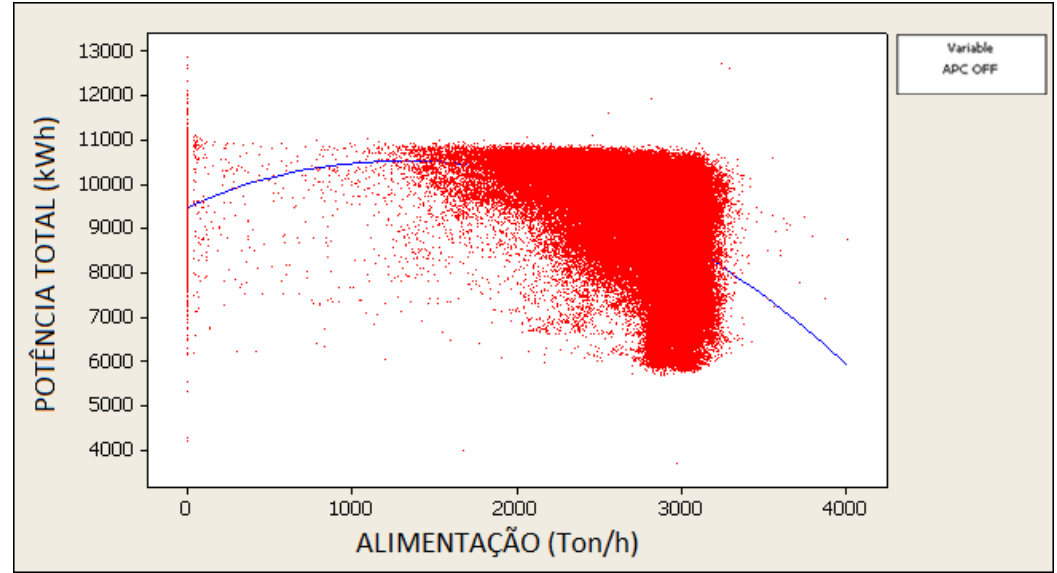

Figura 8 - Curva de potência do moinho e respectivos valores experimentais sem APC.

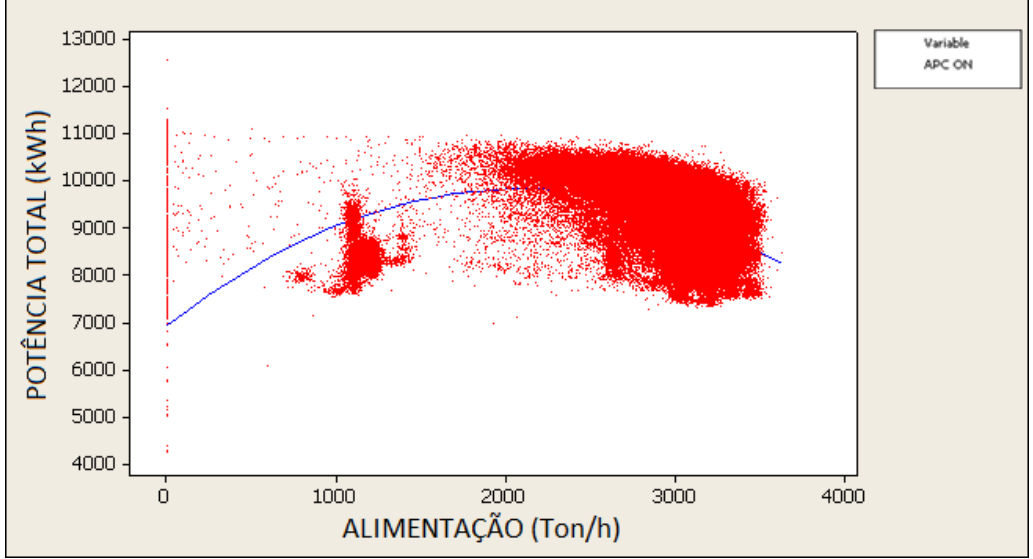

Figura 9 - Curva de potência do moinho e respectivos valores experimentais com APC.

\section{CONCLUSÕES}

A estratégia de controle avançado resultou em um aumento médio de aproximadamente $6 \%$ na taxa de produção e em uma redução de aproximadamente $4 \%$ no valor médio do consumo específico de energia. Adicionalmente, foi verificada uma redução de $8 \%$ no valor médio da porcentagem de partículas com tamanho fora da especificação.

Deve ser ressaltado o fato de que outros aspectos não tangíveis poderiam ser também considerados. Por exemplo, em longo prazo, a redução nos eventos de sobrecarga, contribui de forma direta para reduzir o desgaste mecânico no sistema de acionamento, no revestimento do moinho e no consumo de bolas.

O projeto apresentado neste trabalho resultou em um retorno do investimento realizado inferior a três meses, considerando o volume de produção, o custo de produção e o valor agregado do produto em processamento. 


\section{AGRADECIMENTOS}

Os autores agradecem a Fundação de Amparo à Pesquisa do Estado de Minas Gerais (FAPEMIG) pelos recursos concedidos no Projeto de Participação Coletiva em Evento Científico ou Tecnológico (PCE-00019-13).

\section{REFERÊNCIAS BIBLIOGRÁFICAS}

1. AGUIRRE, L. A. Introdução à identificação de sistemas: técnicas lineares e não lineares aplicadas a sistemas reais. Minas Gerais: Ed. UFMG, 2007.

2. GOUVEIA, R., Lewis, D. G., RODRIGUES, L. A. e GEDRAITE, R. Application of Model-Predictive Control to Multi-Hearth Nickel Reduction Roasters, In: Proceedings of IFACMMM 2009 Workshop on Automation in Mining, Mineral and Metal Industry. October 2009, Vinãs del Mar, Chile.

3. HONEYWELL INTERNATIONAL INC. Profit Controller Concepts Reference Guide, USA. 2008.

4. LAAKSONEN, J.; PYöTSIä, J. Process variability simulated, tested, minimized. In Tech, v.45, n.12, p.68-70, Dec. 1998.

5. MCMILLAN, G. K. Tuning and control loop performance. $3^{\text {rd }}$ ed., Research Triangle Park, NC, ISA - The Instrumentation, Systems and Automation Society, 1994.

6. QIN, S. J., BADGWELL T. A. A Survey of Industrial Model Predictive Control Technology. Control Engineering Practice, Vol. 11, 2003, 733-764.

7. RAMASAMY, M., NARAYANAN, S. S., RAO, D. P. Control of Ball Mill Grinding Circuit Using Model Predictive Control Scheme. - Journal of Process Control, Vol. 15, 2005, 273-283.

8. JKMRC - Julius Kruttschnitt Mineral Research Center. Mineral Comminution Circuits; Their Operation and Optimisation. Australia. 1995. 413p.

9. RESTREPO, A. E., YUZAK, E., RODRIGUES, L. A., AFFONSO, R. e GEDRAITE, R. Considerações práticas acerca da aplicação de sistemas híbridos de controle avançado na indústria de celulose e papel, In: anais do $42^{\circ}$ Congresso Internacional de Celulose e Papel. Outubro 2009, São Paulo, SP.

10. SEBORG, D. E., MELLICHAMP, D. A., EDGAR, T. F. et al. (2005) and DOYLE III, F. J.: Process Dynamics and Control. USA. Wiley, 2011. 